\title{
Defocus Blur Correcting Projector-Camera System
}

\author{
Yuji Oyamada and Hideo Saito \\ Graduate School of Science and Technology, Keio University, \\ 3-14-1 Hiyoshi Kohoku-ku, Yokohama 223-8522, Japan \\ \{charmie, saito\}@ozawa.ics.keio.ac.jp \\ http://www.hvrl.ics.keio.ac.jp
}

\begin{abstract}
To use a projector anytime anywhere, a lot of projectorcamera based approaches have been proposed. In this paper, we focus on a focal correction technique, one of projector-camera based approaches, reduces an effect of defocus blur which occurs when a screen is located out of projector's depth-of-field. We propose a novel method for estimating projector defocus blur on a planar screen without any special measuring images. To estimate a degree of defocus blur accurate, we extract subregion which is well-suited for defocus blur estimation in a projection image and estimate the degree of defocus blur at each extracted region. To remove the degradation caused by the defocus blur, we pre-correct the projection image depends on the degree of defocus blur before projection. Experimental results show that our method can estimate the degree of defocus blur without projecting any special images and pre-corrected image can reduce the effect of the defocus blur.
\end{abstract}

Keywords: Projector-Camera System, Focal Correction, Defocus Blur.

\section{Introduction}

The advantage of a projector is we can easily change size of displayed image on a screen. Over the last decade, projectors have been improved in their quality (e.g., brightness, resolution, contrast, throw-distance, etc.) and now a projector has come to be used for nonconventional usages. Recently, projectors are used for overlaying virtual objects onto real world objects, so that multiple users can experience the Augmented Reality applications at the same time 11. For example, Yotsukura et al. have proposed a system that supports an actor wearing a face mask by using a projector [2]. This system tracks a face mask with infrared LEDs which an actor wears so that the projected image can always be attached onto the face mask surface. Augmented book was proposed by Gupta and Jaynes [3. In this system, a non-textured book is placed on a table and an user flips over the pages. Using a camera, the system tracks the $3 \mathrm{D}$ position of the page and virtual multimedia content, including images and volumetric datasets, is correctly warped. Then a projector projects warped virtual multimedia onto each page at interactive rates. Thus, projection onto moving or volumetric surface 
is state-of-the-art application. However, there are still some difficult problems to project onto moving or volumetric surface. Because projectors are basically designed to project an image onto non-textured and non-colored planar screen which should be located at perpendicular position to projector's lighting direction. When we use a projector in defiance of these limitations, a displayed image is degraded. It is a current challenge to use a projector in arbitrary situation e.g. off-axis projection.

To display an image as if we use a projector at ideal situation, a lot of projector-camera based approaches have been proposed. The idea of these approaches is to consider displayed image degradation as a filter function. By projecting an inverse filtered image, we can avoid this degradation. Recently, focal correction technique which aims to remove the defocus blur has attracted attention. Projectors are designed to have large apertures that lead to narrow depth-of-field.

In this paper, we have extended previous method which aims to estimate shift variant PSF (Point Spread Function) across the display surface [4. We consider this degradation as a shift variant PSF. To estimate shift variant PSF, we interpolate PSFs estimated at extracted regions which are well-suited for PSF estimation. Then we apply Wiener filtering based on the estimated shift variant PSF with a projection image. Finally, we can avoid projector image degradation caused by defocus blur by projecting Wiener filtered image in an experiment.

This paper is organized as follows: In section 2 we discuss related work; section 3 explains preliminaries of our proposed method; section 4 describes the detail of proposed method; section 5 shows experimental results; section 6 concludes this paper with summarizing of our method.

\section{Related Works}

Research on projector-camera based approaches which aim to avoid projector image degradation can be categorized into three types: geometric warping, color compensation and focal correction.

Geometric Warping techniques have been developed to make displayed image rectified under a situation, e.g. slanted projection, projection onto a non-planar surfaces and multiple projectors projection alignment. This technique depends on a shape of projected surface, linear or non-linear. In the case of linear, we can describe a relation between projector image and camera image as a linear function [567]. On the other hand, the relation should be corresponded on a pixel to pixel level 86]. This technique is well discussed by Brown et al. [10].

Radiometric Compensation techniques aim to correct color variation caused by screen color, texture, environmental lighting and light attenuation corresponding to a distance between projector and displaye surface. This approach needs to know color mapping between projector and camera. Compensation techniques for both static [1] and dynamic scene [12] have been proposed. Ashdown et al. have proposed content-dependent compensation technique based on both radiometric model and human visual system [13. Wetzstein et al. have accounted 
for all possible local and global illumination effects by applying the inverse light transport between a light source and a projector 14.

Focal correction technique is to avoid a degradation caused by defocus blur. There are mainly two solutions, multiple projectors method and pre-corrected image projection method. Multiple projectors method has been proposed by Bimber et al. [15]. Each projector is adjusted to different focal plane. First, they rasrerize a fiducial pattern image projection to estimate shift variant PSF which represents a degree of a defocus on a non-planar display. Then, each projector projects an image onto a part of a screen which is located in the each projector's depth-of-field to minimize a degradation caused by defocus blur. Another approach projects a pre-corrected image 1617/8. Brown et al. have focused on projector defocus caused on a planar display. First, they project a fiducial pattern to know an in-focus region in a planar display and estimate a shift variant PSF which represents how much a sub region of a display is blurred from in-focus region. Next, they apply Wiener filtering to an original image based on a shift variant PSF. Projected result of a Wiener filtered image remove projector defocus. Inherently, geometric distortion and shift variant PSF has correlative relationship but it is not usable only itself. Because an effect of a projector defocus depends on a distance between a focal plane of a projector and display surface. To use geometric information for PSF estimation, some assumption is needed as Brown et al. recognized most in-focus region as an exempler region. However, there is no guarantee that a part of a displayed image is in-focus. When a whole image is blurred, this assumption is failing. Zhang and Nayar have proposed a similar method [16. The point of their method is pre-correction algorithm. When a defocus is severe, Wiener filtering usually occurs artifacts. To reduce this artifact, they iterate inverse filtering as defocus is minimized. We have proposed a PSF estimation method which doesn't need to project any fiducial images [18. An original image is used to estimate a shift variant PSF instead of projecting any fiducial pattern image. We compare a displayed image with a projected image (detail is described in Sec4.2)

To apply a real application, projecting sand displaying any fiducial images interrupts an effect of projector based application. In this sense, our previous method is better. However, our PSF estimation algorithm fails depends on a projected image. Because a part of an image is insusceptible to the projector defocus blur.

\section{Preliminaries}

\subsection{Image Degradation and Restoration}

A degraded image can be modeled as the result of a convolution

$$
g(x, y)=f(x, y) * h(x, y)
$$

where $g(x, y), f(x, y)$ and $h(x, y)$ are a degraded image, an original image and PSF respectively. A model of PSF depends on a type of an image degradation. 
In the case of projector defocus blur, it's due to projector's spherical lens. So we can represent a degradation caused by a projector defocus blur as a 2D Gaussian with standard deviation $\sigma$

$$
h(x, y)=\frac{1}{2 \pi \sigma^{2}} \exp \left(-\frac{x^{2}+y^{2}}{2 \sigma^{2}}\right) .
$$

Based on the traditional image processing approaches, we can restore an unknown original image by convolution with an inverse function $h^{-1}(x, y)$ on a degraded image. To apply inverse filtering, we consider a model in the frequency domain. Because convolution in the spatial domain equals to the multiplication in the frequency domain as

$$
G(u, v)=F(u, v) H(u, v),
$$

where $G(u, v), F(u, v)$ and $H(u, v)$ are Fourier transforms of $g(x, y), f(x, y)$ and $h(x, y)$ respectively. When we know a PSF model, we can restore a non-degraded image by applying Wiener filtering, which is one of the popular solutions that minimizes an effect of deconvolved noise. The Wiener filter $H_{\mathrm{W}}$ is modeled as

$$
H_{\mathrm{W}}=\frac{1}{H} \frac{|H|^{2}}{|H|^{2}+\gamma},
$$

where $\gamma$ is the signal-to-noise ratio in power.

\subsection{Shift Variant PSF}

As previously mentioned, projector defocus blur can be represented as a 2D Gaussian PSF. When the projector is located at slanted position to a display surface, a PSF is shift variant, spatially varying across the display surface. To estimate shift variant PSF, the best way is estimation on pixel by pixel but it's not applicable. Instead of pixel by pixel, we apply interpolation of the PSFs estimated at different position.

\section{Proposed Method}

Our system is consists of a projector, a camera and a planar screen. First, we calibrate the camera and the projector to remove the geometric and color distortion as a preprocessing. The proposed method first extract regions which are well-suited to PSF estimation and then estimate shift variant PSF. By using estimated shift variant PSF, we apply Wiener filtering with a projection image. Finally, Wiener filtered image projection can remove the degradation caused by projector defocus blur.

\subsection{Calibration}

As described in previous section, displayed image degradation on a display surface can be geometrically and radiometrically distorted in addition to the defocus 
blur. To avoid image defection factors, geometrical and radiometric distortion, other than projection defocus blur, we apply geometric warping and color compensation with a projection image.

To remove geometric distortion caused by projection, we need to know mapping between camera pixel and projector pixel. In the case of a planar screen, we can model this mapping as a $3 \times 3$ planar perspective transformation matrix homography [5]. To know a homography between camera image plane and projector image plane, we project a chess board pattern and capture a displayed image of projected chess baord pattern. Then, we compute a homography between a projection image and a displayed image by comparing these images. By using the calculated homography, we make a new projection image which can remove geometric distortion.

We need to know color mapping between camera and projector, to correct color distortion. This color mapping can be described as a CTF (Color Transfer Function) 19. A pixel value in the projected image $I_{\mathrm{p}}(x, y)$ is changed to a pixel value in the captured image $I_{\mathrm{C}}(x, y)$ by using calculated CTF $C$,

$$
I_{\mathrm{C}}(x, y)=C\left(I_{\mathrm{p}}(x, y)\right)=\frac{a}{1+\exp ^{-\alpha\left(I_{\mathrm{p}}(x, y)-b\right)}}+k,
$$

where $a, b, k$ and $\alpha$ are parameter. First, we project many uniform color patterns (e.g., dark blue, middle blue and bright blue) with respect to each color component and capture displayed images of projected uniform color patterns. Then, we compute parameters of CTF between a projection image and a displayed image.

Fig 1 is an example how geometric and color distortion work at slanted situation. Fig $1(\mathrm{~d})$ shows how the original image (Fig $1(\mathrm{a})$ is distorted by slanted projection. Geometric distortion is corrected by projecting a warped image as shown in Fig 1(e), but its color is still distorted. Fig 1(c) is geometric warped and color compensated image. By projecting Fig $1(\mathrm{c})$ both geometric and color distortion are corrected as shown in Fig $1(\mathrm{f})$

\subsection{Shift Invariant PSF Estimation}

First, we explain how to estimate a shift invariant (uniform) PSF at a perpendicular projection. The characteristic of a projector defocus blur is that we have a degradation-free image and a PSF model can be represented as a 2D Gaussian as described in Sec 3. So, all we have to do is just specify a value of PSF parameter $\sigma$. Furthermore, we know geometric and color mapping between camera and projector through calibration steps. By using this information, we can predict an image displayed on a surface. We can denote a relation between a displayed image captured by a camera and a predicted image,

$$
f_{c}(x, y)=f_{p}(x, y) * h_{\sigma}(x, y),
$$

where a displayed image, a predicted image and a PSF are $f_{c}(x, y), f_{p}(x, y)$ and $h_{\sigma}(x, y)$. To estimate a PSF on a displayed image, we make multiple blurred images by convolution different PSFs on a predicted image $f_{p}(x, y)$,

$$
g_{p\left(\sigma_{n}\right)}(x, y)=f_{p}(x, y) * h_{\sigma_{n}}(x, y),
$$




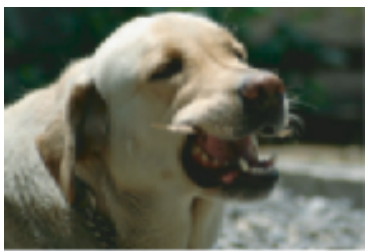

(a) Original image

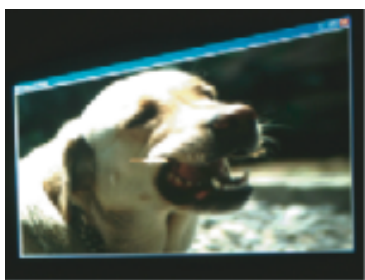

(d) Displayed (a)

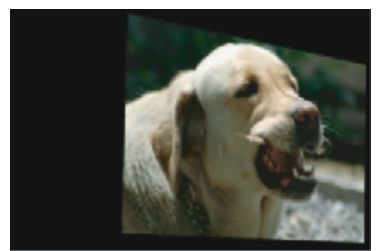

(b) Warped (a)

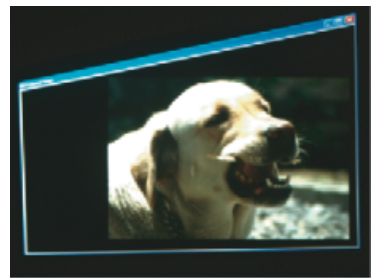

(e) Displayed (b)

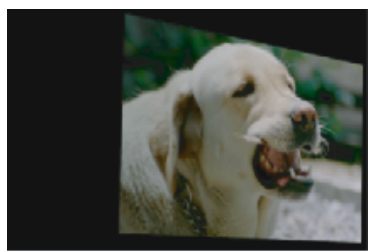

(c) Compensated (b)

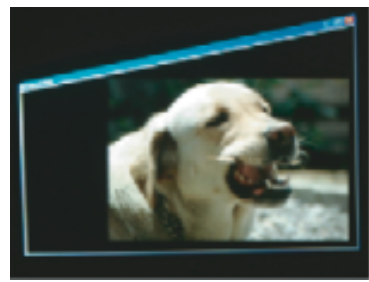

(f) Displayed (c)

Fig. 1. Calibration results: (Top) projection image projected by a projector; (Bottom) displayed image captured by a camera; (Left) non-corrected; (Middle) geometrically warped using homography; (Right) geometrically warped and color compensated using CTF

where $g_{p\left(\sigma_{n}\right)}(x, y)$ represents a blurred image by convolution a 2D Gaussian PSF $h_{\sigma_{n}}(x, y)$ with parameter $\sigma_{n}$. Then we calculate NCC (Normalized Cross Correlation) between a displayed image $f_{c}(x, y)$ and each blurred image $g_{p\left(\sigma_{n}\right)}(x, y)$ and choose the one with highest NCC value. The PSF which makes this chosen image represents a projector defocus on the blurred image $f_{c}(x, y)$.

\subsection{Shift Variant PSF Estimation}

Next, we discuss how we compute a shift variant PSF across a display surface. We assume that a PSF in a small region of a displayed image is uniform. This assumption allows us to apply interpolation of shift invariant PSFs. The point of this estimation method is where we estimate shift invariant PSFs for interpolation. When we estimate a shift invariant PSF, we should carefully choose a small region. For example, when we use a region which has nearly uniform color, that region is ill-suited for PSF estimation. Because it's difficult to know whether an image is blurred by seeing such a region.

First of all, we define both well-suited and ill-suited region for PSF estimation. Basic idea for the definition comes from the method creates an arbitrary focused image proposed by Aizawa et al. 20]. When an image is less textured (like uniform) or has not in-focus region, such an image is not sensitive to projecter defocus blur. So we define a region sensitive to defocus blur as well-suited region for PSF estimation. To extract a well-suited region, we prepare two images, an original image $f(x, y)$ and a blurred image $g(x, y)$ which is result of convolution of PSF on an original image. We divide an original image into four blocks, i.e.: 
left-top, right-top, left-bottom and right-bottom, and apply following processing with each block. We extract a sub region from a divided block and then calculate SAD (Sum of Absolute Difference) value between a sub region $f_{i, j}(x, y)$ and a blurred sub region $g_{i, j}(x, y)$ as,

$$
\mathrm{SAD}_{i, j}=\sum_{y=i}^{i+n} \sum_{x=j}^{j+n}\left|f_{i, j}(x, y)-g_{i, j}(x, y)\right|
$$

where $\mathrm{SAD}_{i, j}$ represents $\mathrm{SAD}$ value between a sub region and a blurred sub region. A sub region with highest $\mathrm{SAD}$ value represents a sub region which is most sensitive to defocus blur.

Fig 2 shows an example. Left image and right image of 2(a) are corresponding to an original image and a blurred image. By comparing sub regions of these images, we can extract well-suited regions. Red framed regions in Fig 2(b) are corresponding to sub regions extracted by proposed method.

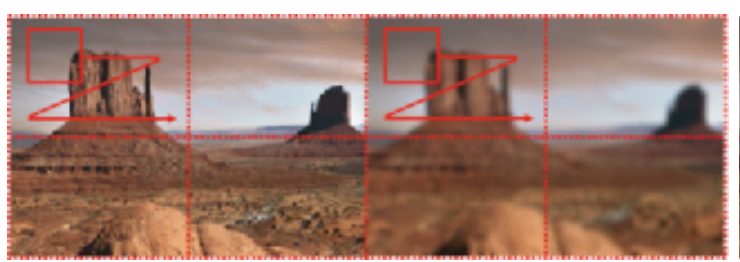

(a) Comparison original and blurred image

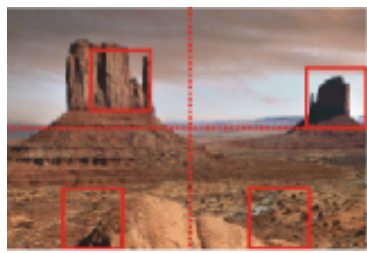

(b) sub-region

Fig. 2. Well-suited region extraction: (a)left and right corresponding to the original image and the blurred image. SAD value is calculated at each sub region; (b)sub region with highest $\mathrm{SAD}$ value is well-suited region.

After Rasterizing this SAD value computation at every block, we have four well-suited regions for PSF estimation. Now, we have four shift invariant PSFs estimated at extracted sub regions. They are defined as $h_{0,0}(x, y), h_{0,1}(x, y)$, $h_{1,0}(x, y), h_{1,1}(x, y)$, from left top to right top. Interpolated PSF's parameter $\sigma$ is calculated as,

$$
\begin{aligned}
h_{i, j}(x, y)= & \left(1-s_{x}\right)\left(1-s_{y}\right) h_{0,0}(x, y)+s_{x}\left(1-s_{y}\right) h_{0,1}(x, y) \\
& +\left(1-s_{x}\right) s_{y} h_{1,0}(x, y)+s_{x} s_{y} h_{1,1}(x, y),
\end{aligned}
$$

where $s_{x}$ and $s_{y}$ are linear interpolation coefficients in the $x$ and $y$ axis.

\subsection{Projection Image Pre-correction}

As previously mentioned, we apply the Wiener filtering (as expressed in Eq4) as an inverse filtering. However, the Wiener filtering is applied to a whole image in the Frequency domain. So, it is not suited to inverse filtering with a shift variant PSF. In stead of shift variant Wiener filtering, we interpolate four Wiener 
filtered images in Spatial domain. First, we compute four PSFs which are corresponding to PSFs at image corners. Next, we make four Wiener filtered images and interpolate these Wiener filtered images as a pre-corrected image. When we project this interpolated image $\tilde{f}(x, y)$, it is degraded by projection as,

$$
\tilde{f}(x, y) * h(x, y)=\left[f(x, y) * h^{-1}(x, y)\right] * h(x, y) \approx f(x, y),
$$

where $\tilde{g}(x, y)$ and $f(x, y)$ represent a displayed result of pre-corrected image and an original image respectively. In theory, a degraded pre-corrected image can become similar to an original image.

\section{Experimental Result}

The proposed method has been tested about PSF estimation and pre-corrected image projection. A projection images are projected by a projector (EPSON ELP7600) which is located in front of a target screen. Displayed images on a screen are captured by a camera (SONY XCDC710CR). The projection image, the extracted region and the displayed image is 960x640, 160x160 and 1024x768 pixels resolution.

\subsection{PSF Estimation}

First, we examine PSF estimation method by using 10 images under the following situations.

- slanted projection (low gradient)

- partially blurred

- wholly blurred

- slanted projection (high gradient)

- partially blurred

- wholly blurred

To evaluate experimental results, we consider the estimated PSF by using fiducial patterns as correct answer. Tb1 1 shows mean error of the estimated PSF between results of proposed method and result of fiducial pattern.

Table 1. Mean error of estimated PSF between proposed method and fiducial pattern

\begin{tabular}{|c|c|c|c|c|}
\hline & \multicolumn{2}{|c|}{ slanted (low gradient) } & \multicolumn{2}{c|}{ slanted (high gradient) } \\
\hline & partially blur & wholly blur & partially blur & wholly blur \\
\hline mean error & 0.55 & 0.88 & 0.87 & 0.97 \\
\hline variance & 0.70 & 0.98 & 1.03 & 0.56 \\
\hline
\end{tabular}

Next, we estimate the shift variant PSF by proposed method. We compare with our previous method [18. Fig 3 shows the experimental results. The estimated PSF by proposed method (as shown in Fig $3(\mathrm{c})$ is increases from left to 
right same as the result of fiducial pattern (as shown in Fig 3(a). Though the error occurs especially at the left side, but it's less than $1.0 \sigma$ value. On the other hand, PSF estimated by previous method has a large margin of error. Especially, it's obviously showed up at the top area of the image. This error caused by using the less textured region to estimate PSF. In this sense, proposed method can work well. Tb 2 shows mean error of the estimated PSF between fiducial pattern and proposed method or previous method. These results show that proposed method can estimate shift variant PSF more than previous method.

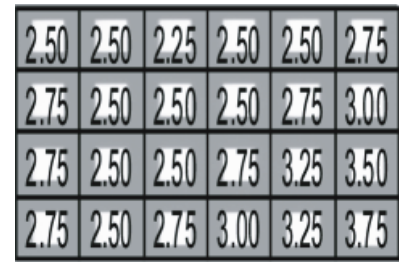

(a) fiducial pattern

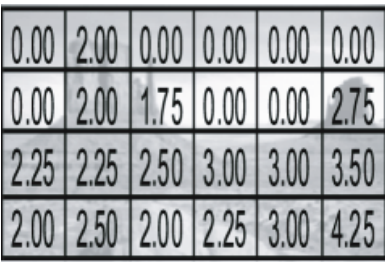

(b) previous method

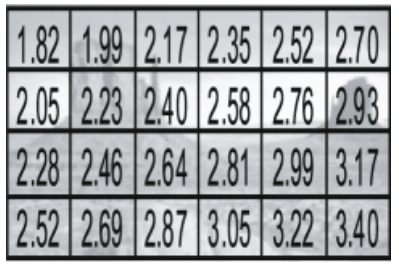

(c) proposed method

Fig. 3. Estimated shift variant PSF: (a) estimated by using fiducial pattern; (b) estimated by previous method; (c) estimated by proposed method

Table 2. PSF estimated by proposed method and previous method [18]

\begin{tabular}{|c|c|c|c|c|}
\hline & \multicolumn{4}{|c|}{ previous method } \\
\hline & \multicolumn{2}{|c|}{ slanted projection (low gradient) } & \multicolumn{2}{|c|}{ slanted projection (high gradient) } \\
\hline & partially blur & wholly blur & partially blur & wholly blur \\
\hline mean error & \begin{tabular}{|l|}
0.83 \\
\end{tabular} & 2.10 & 1.75 & 2.30 \\
\hline variance & 1.08 & 3.46 & 1.35 & 4.23 \\
\hline & \multicolumn{4}{|c|}{ proposed method } \\
\hline & \multicolumn{2}{|c|}{ slanted projection (low gradient) } & \multicolumn{2}{|c|}{ slanted projection (high gradient) } \\
\hline & partially blur & wholly blur & partially blur & wholly blur \\
\hline mean error & \begin{tabular}{|l|l|}
0.93 \\
\end{tabular} & 1.35 & \begin{tabular}{|l|}
1.85 \\
\end{tabular} & 1.27 \\
\hline variance & 0.86 & 1.06 & 6.40 & 1.43 \\
\hline
\end{tabular}

\subsection{Pre-corrected Image Projection}

Next, we test pre-corrected image projection. We compare the proposed method with both non-corrected image and our previous method [18.

By comparing the projected images (Fig 4(a) Fig 4(c) , projected image corrected by the proposed method has most emphasized edge. Especially, tiger's fur and whisker in Fig 4(c) is clearly different. On the other hand, projected image corrected by previous method [18] has two non-corrected regions. The region where is not corrected is the sub region where we fail PSF estimation. Fig 4(d) Fig 4(f) are displayed result of each projected image and Fig 4(h) Fig $4(\mathrm{j})$ are zoomed figures of Fig $4(\mathrm{~d}) \sim$ Fig $4(\mathrm{f})$. By comparing zoomed results, 


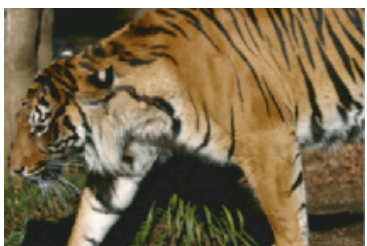

(a) Original image

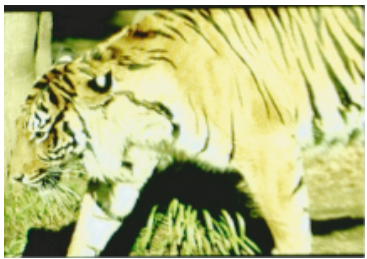

(d) Displayed (a)

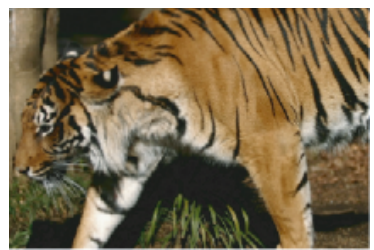

(b) Corrected image by 18

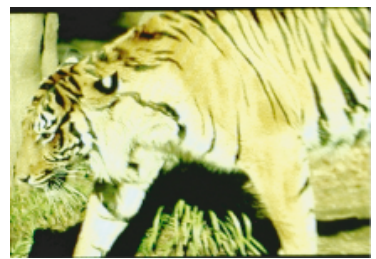

(e) Displayed (b)

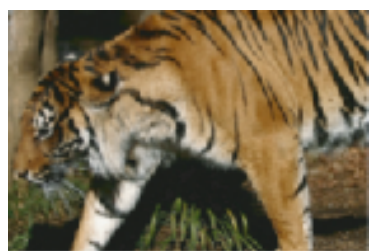

(c) Corrected image by our method

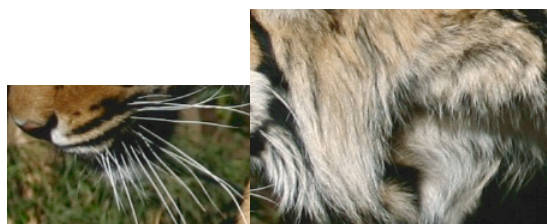

(g) Zoomed (a)

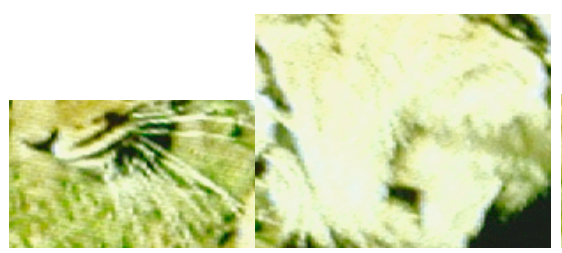

(i) Zoomed (e)

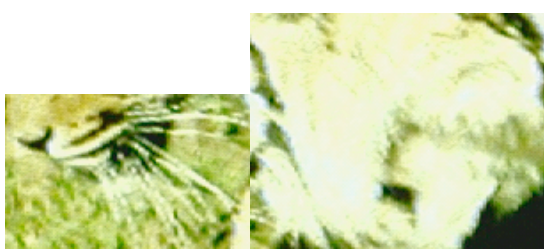

(h) Zoomed (d)

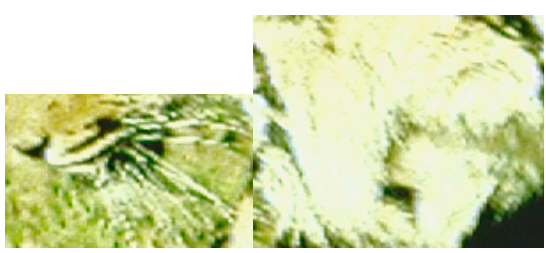

(j) Zoomed (f)

Fig. 4. Pre-corrected image removes the degradation caused by the projector defocus: (Top) projected image ; (Second row)Displayed result of projection; (Third and fourth row) Zoomed images of displayed result

we can see sharpest fur and whisker in the zoomed result of proposed method (as shown in Fig 4(j)]. It means the proposed method can remove the effect of projector defocus more than previous method.

\section{Conclusion}

We extend shift variant PSF estimation method for pre-correction to reduce defocus projection blur without projecting any measuring images. By extracting well-suited region for PSF estimation and applying interpolation of the shift 
invariant PSFs estimated at extracted region, we can estimated shift variant PSF more accurate than previous method. Experimental results show that the our method successfully estimate shift variant PSF, even though the projection image has less-textured and not in-focus region and reduce the effect of the defocus blur.

\section{References}

1. Bimber, O., Raskar, R.: Spatial Augmented Reality Merging Real and Virtual Worlds. A K Peters LTD (2005)

2. Yotsukura, T., Nielsen, F., Binsted, K., Morishima, S., Pinhanez, C.S.: Hypermask: Talking head projected onto real object. The Visual Computer 18(2), 111-120 (2002)

3. Gupta, S., Jaynes, C.: The universal media book: Tracking and augmenting moving surfaces with projected information. In: International Symposium on Mixed and Augmented Reality 2006 (ISMAR 2006), pp. 177-180 (2006)

4. Oyamada, Y., Saito, H.: Estimation of projector defocus blur by extracting texture rich regionin projection image. In: The 16th International Conference in Central Europe on Computer Graphics,Visualization and Computer Vision (WSCG 2008), pp. 153-160 (2008)

5. Chen, H., Sukthankar, R., Wallace, G., Li, K.: Scalable alignment of large-format multi-projector displays using camera homography trees. In: Proceedings of the conference on Visualization 2002 (VIS 2002), pp. 339-346 (2002)

6. Raskar, R., van Baar, J., Willwacher, T., Rao, S.: Quadric transfer for immersive curved screen displays. Computer Graphics Forum 23(3), 451-460 (2004)

7. Johnson, T., Fuchs, H.: Real-time projector tracking on complex geometry using ordinary imagery. In: IEEE International Workshop on Projector-Camera Systems (PROCAMS 2007) (2007)

8. Raskar, R., Brown, M.S., Yang, R., Chen, W.-C., Welch, G., Towles, H., Seales, B., Fuchs, H.: Multi-projector displays using camera-based registration. In: Proceedings of the conference on Visualization 1999 (VIS 1999), pp. 161-168 (1999)

9. Tardif, J.-P., Roy, S., Trudeau, M.: Multi-projectors for arbitrary surfaces without explicit calibration nor reconstruction. In: Proceedings of the Fourth International Conference on 3-D Digital Imaging and Modeling (3DIM 2003), pp. 217-219 (2003)

10. Brown, M., Majumder, A., Yang, R.: Camera-based calibration techniques for seamless multiprojector displays. IEEE Transactions on Visualization and Computer Graphics 11(2), 193-206 (2005)

11. Grossberg, M.D., Peri, H., Nayar, S.K., Belhumeur, P.N.: Making one object look like another: Controlling appearance using a projector-camera system. In: Proceedings of the 2004 IEEE Computer Society Conference on Computer Vision and Pattern Recognition (CVPR 2004), pp. 452-459 (2004)

12. Fujii, K., Grossberg, M.D., Nayar, S.K.: A projector-camera system with real-time photometric adaptation for dynamic environments. In: Proceedings of the 2005 IEEE Computer Society Conference on Computer Vision and Pattern Recognition (CVPR 2005), pp. 814-821 (2005)

13. Ashdown, M., Okabe, T., Sato, I., Sato, Y.: Robust content-dependent photometric projector compensation. In: IEEE International Workshop on Projector-Camera Systems (PROCAMS 2006), p. 6 (2006) 
14. Wetzstein, G., Bimber, O.: Radiometric compensation of global illumination effects with projector-camera systems. In: ACM SIGGRAPH 2006 Research posters (SIGGRAPH 2006), p. 38 (2006)

15. Bimber, O., Emmerling, A.: Multifocal projection: A multiprojector technique for increasing focal depth. IEEE Transactions on Visualization and Computer Graphics 12(4), 658-667 (2006)

16. Zhang, L., Nayar, S.: Projection defocus analysis for scene capture and image display. In: ACM SIGGRAPH 2006 Papers (SIGGRAPH 2006), pp. 907-915 (2006)

17. Brown, M.S., Song, P., Cham, T.-J.: Image pre-conditioning for out-of-focus projector blur. In: Proceedings of the 2006 IEEE Computer Society Conference on Computer Vision and Pattern Recognition (CVPR 2006), pp. 1956-1963 (2006)

18. Oyamada, Y., Saito, H.: Focal pre-correction of projected image for deblurring screen image. In: IEEE International Workshop on Projector-Camera Systems (PROCAMS 2007), pp. 1-8 (2007)

19. Jaynes, C., Webb, S., Steele, R.M.: Camera-based detection and removal of shadows from interactive multiprojector displays. IEEE Transactions on Visualization and Computer Graphics 10(3), 290-301 (2004)

20. Aizawa, K., Kodama, K., Kubota, A.: Producing object-based special effects by fusing multiple differently focused images. IEEE Transactions on Circuits and Systems for Video Technology 10(2), 323-330 (2000) 